\title{
ON A SCHUR COMPLEMENT INEQUALITY FOR THE HADAMARD PRODUCT OF CERTAIN TOTALLY NONNEGATIVE MATRICES*
}

\author{
ZHONGPENG YANG ${ }^{\dagger}$ AND XIAOXIA FENG ${ }^{\ddagger}$
}

\begin{abstract}
Under the entrywise dominance partial ordering, T.L. Markham and R.L. Smith obtained a Schur complement inequality for the Hadamard product of two tridiagonal totally nonnegative matrices. Applying the properties of the Hadamard core of totally nonnegative matrices, the Schur complement inequalities for the Hadamard product of totally nonnegative matrices is obtained, which extends those of T.L. Markham and R.L. Smith for tridiagonal totally nonnegative matrices [T.L. Markham and R.L. Smith. A Schur complement inequality for certain P-matrices. Linear Algebra and its Applications, 281:33-41, 1998.]. This result improves the refinement and range of applications for these inequalities.
\end{abstract}

Key words. Totally nonnegative matrix, Tridiagonal matrix, Hadamard core, Schur complement, Matrix inequality, Hadamard product, SC-closure.

AMS subject classifications. 15A09, 15A45, 15A48.

1. Introduction. Let $\mathbb{R}^{m \times n}, S_{n}^{+}$be the sets of $m \times n$ real and $n \times n$ real symmetric positive definite matrices, respectively. The Löwner partial ordering on positive semidefinite matrices $A$ and $B$ is defined as $A \geq B$ if and only if $A-B$ is positive semidefinite. The Hadamard product of two $m \times n$ matrices $A=\left(a_{i j}\right)$ and $B=\left(b_{i j}\right)$ is denoted by $A \circ B=\left(a_{i j} b_{i j}\right)$.

Let $A \in \mathbb{R}^{n \times n}$ be partitioned as follows

(1.1) $A=\left[\begin{array}{ll}A_{11} & A_{12} \\ A_{21} & A_{22}\end{array}\right]$, where $A_{11} \in \mathbb{R}^{k \times k}$, and $A_{22} \in \mathbb{R}^{(n-k) \times(n-k)}$ is invertible.

The matrix

$$
A / A_{22}=A_{11}-A_{12} A_{22}^{-1} A_{21}
$$

*Received by the editors on August 1, 2010. Accepted for publication on February 13, 2011. Handling Editor: Michael Tsatsomeros.

${ }^{\dagger}$ Department of Mathematics, Putian University, Putian, Fujian, 351100, P.R. China (yangzhongpeng@126.com). Supported by Hercynian building for the colleges and universities service in Fujian province (No 2008HX03), and the Education Committee of Fujian Province (No JA08196).

${ }^{\ddagger}$ Department of Mathematics, Zhangzhou Normal University, Zhangzhou, Fujian, 363000, P.R. China (fengxiaoxia0110@126.com). Supported by the Natural Science Foundation of Fujian Province (No 2010J01018), and the Education Committee of Fujian Province (No JA09167). 
is the Schur complement of $A_{22}$ in $A$.

In the Löwner partial ordering, T.L. Markham and R.L. Smith presented a Schur complement inequality for the Hadamard product of real symmetric positive definite matrices partitioned as in (1.1) (see [10, Theorem 1.2]), where the Schur complement is determined by (1.2) as follows

$$
(A \circ B) /\left(A_{22} \circ B_{22}\right) \geq A / A_{22} \circ B / B_{22}, \quad A, B \in S_{n}^{+} .
$$

If all the minors of $A \in \mathbb{R}^{m \times n}$ are nonnegative (positive), we call $A$ as totally nonnegative (positive) matrix, written $A \in T N_{m, n}\left(T P_{m, n}\right)$ (see [2]). When $m=n$, we simply write as $T N_{m, n}=T N_{n}$. A.S. Crans, S.M. Fallat, and C.R. Johnson [2] pointed out that this class arises in a long history of applications, and it has enjoyed increasing recent attention (see [1], [2], [4], [6], [10], [14]). It plays a crucial role in Economics, and has great influence on other branches of Mathematics.

Under the entrywise dominance partial ordering (namely, when every element of $A-B$ is nonnegative, written $A \geq B$ ), T.L. Markham and R.L. Smith proved:

Proposition 1.1. [10, Theorem 2.1] Let $A, B \in T N_{n}$ both be tridiagonal and partitioned as in (1.1). If $A_{22}, B_{22}$ and $A_{22} \circ B_{22}$ are invertible, then the inequality (1.3) holds.

The matrices in the following Example 1.2 come from [9] and [10] as follows

Example 1.2. Let

$$
A=\left(a_{i j}\right)=\left[\begin{array}{ccc}
8 & 12 & 13.05 \\
4 & 7 & 8 \\
0 & 2 & 3
\end{array}\right], \quad B=\left(b_{i j}\right)=\left[\begin{array}{ccc}
1 & 1 & 0 \\
21 & 24 & 14 \\
90.03 & 105 & 70
\end{array}\right] \text {. }
$$

By [9] and [10], we know that both $A$ and $B$ are totally nonnegative matrices. In 1970, T.L. Markham proved $A \circ B \notin T N_{3}$ (see [9]), and $A$ and $B$ satisfy (1.1) with $A_{11}=8, B_{11}=1$, and $(A \circ B) /\left(A_{22} \circ B_{22}\right)=-10, A / A_{22} \circ B / B_{22}=(2 / 25)(1 / 50)=$ $(1 / 25)^{2}$. Thus

$$
(A \circ B) /\left(A_{22} \circ B_{22}\right)<A / A_{22} \circ B / B_{22} .
$$

Therefore, the inequality (1.3) does not hold for the matrices $A$ and $B$ that are not tridiagonal totally nonnegative ones.

Based on the above fact, T.L. Markham and R.L. Smith indicated that it is clear we cannot hope to improve [10, Theorem 2.1] (Proposition 1.1 in this paper).

When $A \in \mathbb{R}^{m \times n}, A[\alpha \mid \beta]$ is the submatrix of $A$ lying in the rows indexed by $\alpha \subset\langle m\rangle=\{1,2, \ldots, m\}$ and columns indexed by $\beta \subset\langle n\rangle$, its complementary sub- 
matrix is written by $A(\alpha \mid \beta)$, and $A[\alpha \mid \alpha]=A[\alpha], A(\alpha \mid \alpha)=A(\alpha) . A[\alpha \mid \beta)$ is the submatrix of $A$ lying in the rows indexed by $\alpha$ and deleting the columns indexed by $\beta$, its complementary submatrix is $A(\alpha \mid \beta]$. For a given index set $\alpha$, there exists a permutation matrix $P$ such that the matrix $A$ partitioned as (1.1) has the following form

$$
P^{T} A P=\left[\begin{array}{cc}
A[\alpha] & A[\alpha \mid \alpha) \\
A(\alpha \mid \alpha] & A(\alpha)
\end{array}\right] \in \mathbb{R}^{n \times n}, \alpha \subset\langle n\rangle,|\alpha|=k,
$$

when $A(\alpha)$ is invertible, write the Schur complement of $A(\alpha)$ in $A$ as

$$
P^{T} A P /\left(P^{T} A P\right)_{22}=A / A(\alpha)=A[\alpha]-A[\alpha \mid \alpha) A(\alpha)^{-1} A(\alpha \mid \alpha],
$$

where $P^{T} A P /\left(P^{T} A P\right)_{22}$ is the Schur complement of $P^{T} A P$ in (1.4) defined by (1.2).

Corresponding to (1.2), F. Zhang pointed out that Schur complements can be formed with respect to any nonsingular submatrix, not just a leading principal submatrix (see [16, p. 20]).

Obviously, when $\alpha=\{1,2, \ldots, k\}$ (i.e., the permutation matrix $P$ in (1.4) is the identity one), the Schur complement $A / A_{22}$ determined by (1.2) is a special case of $A / A(\alpha)$ determined by $(1.5)$.

Under the Löwner partial ordering, B. Wang and F. Zhang [11, Theorem 2] proved a general form of the inequality (1.3) as follows

$$
(A \circ B) /(A(\alpha) \circ B(\alpha)) \geq A / A(\alpha) \circ B / B(\alpha), \quad \alpha \subset\langle n\rangle, \quad A, B \in S_{n}^{+}(n) .
$$

Since the positive property of the class $S_{n}^{+}$is invariant under permutation similarity, and $S_{n}^{+}$is closed under the Hadamard product of real symmetric positive definite matrices (see [8]), the authors of [11], [12], [13], and [15] studied the situation of the partitioned block corresponding to $\alpha=\{1,2, \ldots, k\}$, then by the permutation similarity (1.4), the same conclusion for the general Schur complement $A / A(\alpha)$ defined by (1.5) can be derived.

In 1970, T.L. Markham pointed out that the Hadamard product of the totally nonnegative matrices is not closed in general [9] (also [8] and [16]). From [16, p. 127], we know that the class $T N_{n}$ of totally nonnegative matrices does not have the invariant property under the permutation similarity. Thus, similar to [10], our research for $T N_{n}$ shall carry through under the partitioned form (1.1) in the following discussion.

If $A / A(\alpha) \in \Omega$ for an arbitrary $A \in \Omega$, then the class $\Omega$ is called to be $\alpha S C$ closed (see [8] and [16]); if $\Omega$ is $\alpha$ SC-closed for all $\alpha$, then $\Omega$ is $S C$-closed ([16, p. 111]). By [5], [8], [10], [11], [13] and [16], the discussion for the class with the SCclosure property is very convenient. C.R. Johnson and R.L. Smith (see [16, Chapter 
4]) pointed out that although the totally positive matrix class $T P_{n}$ is $\alpha$ SC-closed for some index sets $\alpha$, but $T P_{n}$ is not SC-closed in general.

The definition of the Hadamard core for totally nonnegative matrices was given in [2] as follows

$$
C T N_{m, n}=\left\{A \in T N_{m, n}: B \in T N_{m, n} \Rightarrow A \circ B \in T N_{m, n}\right\} .
$$

When $m=n, C T N_{m, n}$ is abbreviated as $C T N_{n}$.

By applying the properties of the Hadamard core for totally nonnegative matrices, we obtained a new lower bound for the determinant of the Hadamard product of two totally nonnegative matrices (see [14]).

Applying the properties of the Hadamard core for totally nonnegative matrices, we obtain the Schur complement inequalities for the Hadamard product of totally nonnegative matrices, which extend the results of T.L. Markham and R.L. Smith. Therefore, the restriction of tridiagonal totally nonnegative matrices in $[10$, Theorem 2.1] can be generalized, and the accuracy of the inequalities can be improved.

2. Preliminaries. The Cauchy-Binet formula (see [7, Section 0.8.7, p. 22]) will be used in the following discussion: when $A \in \mathbb{R}^{m \times t}, B \in \mathbb{R}^{t \times n}$, then

$$
\operatorname{det}(A B)[\alpha \mid \beta]=\sum_{\gamma} \operatorname{det} A[\alpha \mid \gamma] \operatorname{det} B[\gamma \mid \beta], \quad \alpha \subset\langle m\rangle, \quad \beta \subset\langle n\rangle
$$

If there exist lower, upper triangular matrices $L, U$ such that $A=L U$, then the matrix $A$ is said to have $L U$-decomposition. The $L U$-decomposition plays an important role in the discussion of totally nonnegative matrices.

By the Cauchy-Binet formula (2.1), [1, Theorem 3.5] or [6, Theorem 5.5], we have

Lemma 2.1. Let $A \in \mathbb{R}^{n \times n}$, then $A \in T N_{n}$ if and only if there are lower and upper triangular matrices $A_{L}, A_{U} \in T N_{n}$ such that $A=A_{U} A_{L}$.

Although we know that $T N_{n}$ is not SC-closed from [16, p. 127], the following Lemma 2.2 indicates that $T N_{n}$ is $\alpha$ SC-closed, where $\alpha=\{1,2, \ldots, k\}$ and $A_{22}=$ $A(\alpha)$ is invertible, $1 \leq k \leq n-1$. This is an important tool in our paper.

Though T. Ando discussed the case for totally positive matrices, by applying the similar method, we can derive the following Lemma 2.2 from [1, Theorem 3.9].

Lemma 2.2. Let $A \in T N_{n}, \alpha=\{1,2, \ldots, k\}$ and $A(\alpha)$ be invertible, then $A / A(\alpha) \in T N_{k}$. 
TheOREM 2.3. Let $A \in T N_{n}, \alpha=\{1,2, \ldots, k\}$ and $A(\alpha)$ be invertible, then

$$
\widehat{A}=\left[\begin{array}{cc}
A[\alpha \mid \alpha) A(\alpha)^{-1} A(\alpha \mid \alpha] & A[\alpha \mid \alpha) \\
A(\alpha \mid \alpha] & A(\alpha)
\end{array}\right] \in T N_{n} .
$$

Proof. According to Lemma 2.1, assume that the LU-decomposition of $A$ is

$$
\begin{aligned}
A & =\left[\begin{array}{cc}
A[\alpha] & A[\alpha \mid \alpha) \\
A(\alpha \mid \alpha] & A(\alpha)
\end{array}\right] \\
& =\left[\begin{array}{cc}
A_{U}[\alpha] A_{L}[\alpha]+A_{U}[\alpha \mid \alpha) A_{L}(\alpha \mid \alpha] & A_{U}[\alpha \mid \alpha) A_{L}(\alpha) \\
A_{U}(\alpha) A_{L}(\alpha \mid \alpha] & A_{U}(\alpha) A_{L}(\alpha)
\end{array}\right]=A_{U} A_{L},
\end{aligned}
$$

where $A_{U}=\left[\begin{array}{cc}A_{U}[\alpha] & A_{U}[\alpha \mid \alpha) \\ 0 & A_{U}(\alpha)\end{array}\right], A_{L}=\left[\begin{array}{cc}A_{L}[\alpha] & 0 \\ A_{L}(\alpha \mid \alpha] & A_{L}(\alpha)\end{array}\right] \in T N_{n}$.

Furthermore, from (1.1), (2.2), and Lemma 2.2, it follows that

$$
A / A(\alpha)=A[\alpha]-A[\alpha \mid \alpha) A(\alpha)^{-1} A(\alpha \mid \alpha]=A_{U}[\alpha] A_{L}[\alpha] \in T N_{k}
$$

By (2.2) and (2.3), we have

$$
A[\alpha \mid \alpha) A(\alpha)^{-1} A(\alpha \mid \alpha]=A[\alpha]-A / A(\alpha)=A_{U}[\alpha \mid \alpha) A_{L}(\alpha \mid \alpha] .
$$

Moreover, from (1.1) and (2.2), we have $A[\alpha \mid \alpha)=A_{U}[\alpha \mid \alpha) A_{L}(\alpha), A(\alpha \mid \alpha]=$ $A_{U}(\alpha) A_{L}(\alpha \mid \alpha], A(\alpha)=A_{U}(\alpha) A_{L}(\alpha)$, thus from (2.4), it follows that

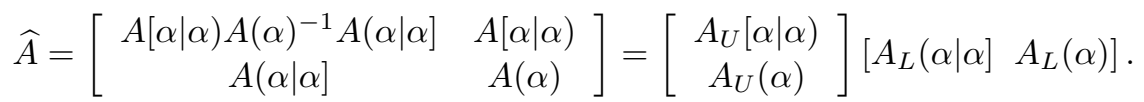

Thus by $A_{U}, A_{L} \in T N_{n}$ again, we know

$$
\left[\begin{array}{c}
A_{U}[\alpha \mid \alpha) \\
A_{U}(\alpha)
\end{array}\right] \in T N_{n, n-k}, \quad\left[A_{L}(\alpha \mid \alpha] A_{L}(\alpha)\right] \in T N_{n-k, n}
$$

thus $\widehat{A} \in T N_{n}$ through (2.1).

The foundations (2.3) and (2.4) in the proof of Theorem 2.3 stem from the discussion of T. Ando for totally positive matrices (see [1, Theorem 3.9] and its proof).

[3, Theorem 15] states "Let $A$ be $n \times n$ totally nonnegative matrix with $\operatorname{det} A(1) \neq$ 0 . Then $A-x E_{11}$ is totally nonnegative for all $x \in\left[0, \frac{\operatorname{det} A}{\operatorname{det} A(1)}\right]$, where $E_{11}=$ $\operatorname{diag}(1,0, \ldots, 0) "$.

From the proof of [3, Theorem 15], we have

$$
A-x E_{11} \in T N_{n} \text { if and only if } A-\frac{\operatorname{det} A}{\operatorname{det} A(1)} E_{11} \in T N_{n}, A \in T N_{n}, \operatorname{det} A(1) \neq 0 .
$$


If let $\alpha=\{1\}$ in Theorem 2.3, by the Schur Complement property [16], we have $A / A(1)=\frac{\operatorname{det} A}{\operatorname{det} A(1)}$, thus $A[1 \mid 1) A(1)^{-1} A(1 \mid 1]=a_{11}-\frac{\operatorname{det} A}{\operatorname{det} A(1)}$.

Furthermore, from Theorem 2.3,

$$
\widehat{A}=\left[\begin{array}{cc}
A[1 \mid 1) A(1)^{-1} A(1 \mid 1] & A[1 \mid 1) \\
A(1 \mid 1] & A(1)
\end{array}\right]=A-\frac{\operatorname{det} A}{\operatorname{det} A(1)} E_{11} \in T N_{n} .
$$

Thus by (2.5), it follows that [3, Theorem 15] can be obtained by Theorem 2.3.

From [10, Section 3], [2, Theorem 2.6] and Theorem 2.3, it follows that

LEMmA 2.4. If the tridiagonal matrix $A \in T N_{n}$, then $\widehat{A} \in C T N_{n}$.

According to [2, Corollary 3.5], we can obtain

Lemma 2.5. Let $A=\left(a_{i j}\right) \in T N_{3}$, then $A \in C T N_{3}$ if and only if

$$
\begin{aligned}
& a_{11} a_{22} a_{33}+a_{31} a_{12} a_{23} \geq a_{11} a_{23} a_{32}+a_{21} a_{12} a_{33}, \\
& a_{11} a_{22} a_{33}+a_{21} a_{13} a_{32} \geq a_{11} a_{23} a_{32}+a_{21} a_{12} a_{33} .
\end{aligned}
$$

3. Main results. Like [10], the matrix inequalities in this section will be given under the entrywise dominance partial ordering.

Theorem 3.1. Let $A, B \in T N_{n}, \alpha=\{1,2, \ldots, k\}, A(\alpha), B(\alpha)$ and $A(\alpha) \circ B(\alpha)$ be all invertible. If $\widehat{A} \in C T N_{n}$, then

$$
\begin{aligned}
(A \circ B) /(A(\alpha) \circ B(\alpha)) & \geq A / A(\alpha) \circ B[\alpha]+A[\alpha] \circ B / B(\alpha)-A / A(\alpha) \circ B / B(\alpha) \\
& \geq A / A(\alpha) \circ B[\alpha] \geq A / A(\alpha) \circ B / B(\alpha),
\end{aligned}
$$

$$
\begin{aligned}
(A \circ B) /(A(\alpha) \circ B(\alpha)) & \geq A / A(\alpha) \circ B[\alpha]+A[\alpha] \circ B / B(\alpha)-A / A(\alpha) \circ B / B(\alpha) \\
& \geq A[\alpha] \circ B / B(\alpha) \geq A / A(\alpha) \circ B / B(\alpha) .
\end{aligned}
$$

Proof. Applying (1.1), (1.2) and Theorem 2.3, we have $\widehat{A} \in T N_{n}$ and

$$
\widehat{B}=\left[\begin{array}{cc}
B[\alpha \mid \alpha) B(\alpha)^{-1} B(\alpha \mid \alpha] & B[\alpha \mid \alpha) \\
B(\alpha \mid \alpha] & B(\alpha)
\end{array}\right]=\left[\begin{array}{cc}
B[\alpha]-B / B(\alpha) & B[\alpha \mid \alpha) \\
B(\alpha \mid \alpha] & B(\alpha)
\end{array}\right] \in T N_{n} .
$$

Since $\widehat{A} \in C T N_{n}$ and the definition of the Hadamard core given by (1.6), then

$$
\widehat{A} \circ \widehat{B}=\left[\begin{array}{cc}
(A[\alpha]-A / A(\alpha)) \circ(B[\alpha]-B / B(\alpha)) & A[\alpha \mid \alpha) \circ B[\alpha \mid \alpha) \\
A(\alpha \mid \alpha] \circ B(\alpha \mid \alpha] & A(\alpha) \circ B(\alpha)
\end{array}\right] \in T N_{n} .
$$


Thus using (1.1), (1.2), and Lemma 2.2 again, it follows that

$$
\begin{aligned}
(\widehat{A} \circ \widehat{B}) / & (A(\alpha) \circ B(\alpha)) \\
= & (A[\alpha]-A / A(\alpha)) \circ(B[\alpha]-B / B(\alpha)) \\
& -(A[\alpha \mid \alpha) \circ B[\alpha \mid \alpha))(A(\alpha) \circ B(\alpha))^{-1}(A(\alpha \mid \alpha] \circ B(\alpha \mid \alpha]) \\
= & A[\alpha] \circ B[\alpha]-(A[\alpha \mid \alpha) \circ B[\alpha \mid \alpha))(A(\alpha) \circ B(\alpha))^{-1}(A(\alpha \mid \alpha] \circ B(\alpha \mid \alpha]) \\
& -(A[\alpha] \circ B / B(\alpha)+A / A(\alpha) \circ B[\alpha]-A / A(\alpha) \circ B / B(\alpha)) \\
= & (A \circ B) /(A(\alpha) \circ B(\alpha)) \\
& -(A[\alpha] \circ B / B(\alpha)+A / A(\alpha) \circ B[\alpha]-A / A(\alpha) \circ B / B(\alpha)) .
\end{aligned}
$$

Note that $(\widehat{A} \circ \widehat{B}) /(A(\alpha) \circ B(\alpha)) \in T N_{k}$ by Lemma 2.2 , hence, we know it is entrywise nonnegative. Therefore

$(3.3)(A \circ B) /(A(\alpha) \circ B(\alpha)) \geq A / A(\alpha) \circ B[\alpha]+A[\alpha] \circ B / B(\alpha)-A / A(\alpha) \circ B / B(\alpha)$.

By (2.1) and (2.4), we know that both

$A[\alpha \mid \alpha) A(\alpha)^{-1} A(\alpha \mid \alpha]=A[\alpha]-A / A(\alpha)$ and $B[\alpha \mid \alpha) B(\alpha)^{-1} B(\alpha \mid \alpha]=B[\alpha]-B / B(\alpha)$

are totally nonnegative matrices. From Lemma 2.2, we have $A / A(\alpha), B / B(\alpha) \in T N_{k}$. Since $A[\alpha], B[\alpha] \in T N_{k}$, thus from (1.2) and (2.4), it follows that

$$
\begin{aligned}
A / A(\alpha) \circ B[\alpha]+A[\alpha] \circ B / B(\alpha)-A / A(\alpha) \circ B / B(\alpha) \\
\quad=A / A(\alpha) \circ B[\alpha]+(A[\alpha]-A / A(\alpha)) \circ B / B(\alpha) \\
\quad \geq A / A(\alpha) \circ B[\alpha] \\
\quad=A / A(\alpha) \circ\left(B / B(\alpha)+B[\alpha \mid \alpha) B(\alpha)^{-1} B(\alpha \mid \alpha]\right),
\end{aligned}
$$

so (3.1) can be obtained by (3.3).

In the same way, since

$$
\begin{aligned}
A / A & (\alpha) \circ B[\alpha]+A[\alpha] \circ B / B(\alpha)-A / A(\alpha) \circ B / B(\alpha) \\
& =A[\alpha] \circ B / B(\alpha)+A / A(\alpha) \circ(B[\alpha]-B / B(\alpha)) \\
& \geq A[\alpha] \circ B / B(\alpha) \\
& =A / A(\alpha) \circ B / B(\alpha)+\left(A[\alpha \mid \alpha) A(\alpha)^{-1} A(\alpha \mid \alpha]\right) \circ B / B(\alpha),
\end{aligned}
$$

using (3.3) again, then (3.2) holds. $\square$

Theorem 3.2. Let $A, B \in T N_{n}, \alpha=\{1,2, \ldots, k\}$, and $A(\alpha), B(\alpha)$ and $A(\alpha) \circ$ $B(\alpha)$ be invertible. If $\widehat{B} \in C T N_{n}$, then the inequalities (3.1) and (3.2) hold. 
Proof. Because the Hadamard product of matrices is commutative, and thus by the proof of Theorem 3.1, we know the conclusions of Theorem 3.2 hold.

By using Theorems 3.1 and 3.2, we have the following result.

Theorem 3.3. Let $A, B \in T N_{n}, \alpha=\{1,2, \ldots, k\}$, simultaneously, $A(\alpha), B(\alpha)$ and $A(\alpha) \circ B(\alpha)$ are all invertible. If $\widehat{A} \in C T N_{n}$ or $\widehat{B} \in C T N_{n}$, then both (3.1) and (3.2) hold.

Corollary 3.4. Let $A, B \in T N_{n}, \alpha=\{1,2, \ldots, k\}, A(\alpha), B(\alpha)$ and $A(\alpha) \circ B(\alpha)$ be all invertible. If $A$ or $B$ is tridiagonal, then the inequalities (3.1) and (3.2) hold.

Proof. When $A \in T N_{n}$ and is tridiagonal, by Lemma 2.4, we have $\widehat{A} \in C T N_{n}$, furthermore, it follows that the inequalities (3.1) and (3.2) hold by Theorem 3.1. In the same way, when $B \in T N_{n}$ and is tridiagonal, the conclusions hold from Theorem 3.2 .

4. Some remarks. By Corollary 3.4, we know the assumptions of [10, Theorem 2.1] (Proposition 1.1 in this paper) are a special cases of Theorems 3.1-3.3. Thus the inequality (1.3) derived by T.L. Markham and R.L. Smith can be strengthened and improved by (3.1) and (3.2).

Example 4.1. Let $A=\left[\begin{array}{ccc}8 & 4 & 1 \\ 3 & 2 & 1 \\ 1 & 1 & 1\end{array}\right], B=\left[\begin{array}{ccc}10 & 8 & 3 \\ 6 & 7 & 3 \\ 1 & 2 & 1\end{array}\right]$

There are totally nonnegative matrices $A_{U}=\left[\begin{array}{lll}1 & 3 & 1 \\ 0 & 1 & 1 \\ 0 & 0 & 1\end{array}\right], A_{L}=\left[\begin{array}{lll}1 & 0 & 0 \\ 2 & 1 & 0 \\ 1 & 1 & 1\end{array}\right]$, and $B_{U}=\left[\begin{array}{lll}1 & 2 & 3 \\ 0 & 1 & 3 \\ 0 & 0 & 1\end{array}\right], B_{L}=\left[\begin{array}{lll}1 & 0 & 0 \\ 3 & 1 & 0 \\ 1 & 2 & 1\end{array}\right]$ such that $A=A_{U} A_{L}, B=B_{U} B_{L}$, thereby, we have $A, B \in T N_{3}$ by Lemma $2.1\left(A \in T N_{3}\right.$ can also be obtained from [14, Example 3.6]).

Let $\alpha=\{1,2\}$. Then $A(\alpha)=B(\alpha)=A(\alpha) \circ B(\alpha)=1$ are all invertible. According to (1.1) and Theorem 2.3, it follows that

$$
\widehat{A}=\left[\begin{array}{cc}
A[\alpha \mid \alpha) A(\alpha)^{-1} A(\alpha \mid \alpha] & A[\alpha \mid \alpha) \\
A(\alpha \mid \alpha] & A(\alpha)
\end{array}\right]=\left[\begin{array}{ccc}
1 & 1 & 1 \\
1 & 1 & 1 \\
1 & 1 & 1
\end{array}\right] \in T N_{3}
$$

For an arbitrary matrix $C \in T N_{3}$, by means of $\widehat{A} \circ C=C \in T N_{3}$ and (1.4), we get $\widehat{A} \in C T N_{3}$. Thus the inequalities (3.1) and (3.2) hold by applying Theorem 3.1. 
Since $A, B\left(\in T N_{3}\right)$ are not tridiagonal, they do not satisfy the hypothesis of [10, Theorem 2.1]. This indicates that the applicable range of the results in our paper is broader than the one in [10], or the restriction that $A, B \in T N_{n}$ are all tridiagonal can be extended.

Thus from (1.2), (3.1) and (3.2), we get

$$
\begin{aligned}
&(A \circ B) /(A(\alpha) \circ B(\alpha)) \\
&= {\left[\begin{array}{cc}
77 & 26 \\
15 & 8
\end{array}\right] } \\
& \geq A / A(\alpha) \circ B[\alpha]+A[\alpha] \circ B / B(\alpha)-A / A(\alpha) \circ B / B(\alpha)=\left[\begin{array}{cc}
77 & 26 \\
15 & 8
\end{array}\right] \\
& \geq A / A(\alpha) \circ B[\alpha]=\left[\begin{array}{cc}
70 & 24 \\
12 & 7
\end{array}\right] ; \\
&(A \circ B) /(A(\alpha) \circ B(\alpha)) {\left[\begin{array}{cc}
77 & 26 \\
15 & 8
\end{array}\right] } \\
& \quad \geq A / A(\alpha) \circ B[\alpha]+A[\alpha] \circ B / B(\alpha)-A / A(\alpha) \circ B / B(\alpha)=\left[\begin{array}{cc}
77 & 26 \\
15 & 8
\end{array}\right] \\
& \quad \geq A[\alpha] \circ B / B(\alpha)=\left[\begin{array}{cc}
56 & 8 \\
9 & 2
\end{array}\right] .
\end{aligned}
$$

These lower bounds for $(A \circ B) /(A(\alpha) \circ B(\alpha))$ are better than the one $A / A(\alpha) \circ$ $B / B(\alpha)=\left[\begin{array}{cc}49 & 6 \\ 6 & 1\end{array}\right]$ given by $(1.3)$.

For $A=\left(a_{i j}\right)$ in Example 4.1, we have $a_{11} a_{22} a_{33}+a_{21} a_{13} a_{32}=19<20=$ $a_{11} a_{23} a_{32}+a_{21} a_{12} a_{33}$, thereby, we know $A \notin C T N_{3}$ by Lemma 2.5 .

Easily, our assumption is the same as the one of [10, Theorem 2.1]. Theorems 3.1-3.3 and Example 4.1 show that the inequality (1.3) for the Hadamard product of tridiagonal totally nonnegative matrices can be improved on the refinement of inequalities and their applicable range.

ExAMPLE 4.2. Let $C=\left(c_{i j}\right)=\left[\begin{array}{lll}9 & 6 & 3 \\ 6 & 8 & 6 \\ 3 & 6 & 9\end{array}\right]$.

There are totally nonnegative matrices $C_{U}=\left[\begin{array}{lll}2 & 2 & 1 \\ 0 & 2 & 2 \\ 0 & 0 & 3\end{array}\right], C_{L}=\left[\begin{array}{lll}2 & 0 & 0 \\ 2 & 2 & 0 \\ 1 & 2 & 3\end{array}\right]$ 
such that $C=C_{U} C_{L}$, so $C \in T N_{3}$ by Lemma 2.1. And

$c_{11} c_{22} c_{33}+c_{31} c_{12} c_{23}=c_{11} c_{22} c_{33}+c_{21} c_{13} c_{32}=756 \geq c_{11} c_{23} c_{32}+c_{21} c_{12} c_{33}=648$, then $C \in C T N_{3}$ by Lemma 2.5 .

Let $\alpha=\{1\}$, by (1.1) and Theorem 2.3, we get

$$
\widehat{C}=\left[\begin{array}{cc}
C[\alpha \mid \alpha) C(\alpha)^{-1} C(\alpha \mid \alpha] & C[\alpha \mid \alpha) \\
C(\alpha \mid \alpha] & C(\alpha)
\end{array}\right]=\left[\begin{array}{ccc}
5 & 6 & 3 \\
6 & 8 & 6 \\
3 & 6 & 9
\end{array}\right]=\left(\widehat{c}_{i j}\right) \in T N_{3} .
$$

By $\widehat{c}_{11} \widehat{c}_{22} \widehat{c}_{33}+\widehat{c}_{31} \widehat{c}_{12} \widehat{c}_{23}=468<\widehat{c}_{11} \widehat{c}_{23} \widehat{c}_{32}+\widehat{c}_{21} \widehat{c}_{12} \widehat{c}_{33}=504$ and Lemma 2.5, we obtain $\widehat{C} \notin C T N_{3}$.

Corresponding to [10, Theorem 2.1] (Proposition 1.1 in our paper), if we add the condition " $\widehat{A} \in C T N_{n}$ " to our conclusions, from Lemma 2.4, we know that the additive condition is naturally satisfied when $A \in T N_{n}$ is tridiagonal. However, the situation is very different for general totally nonnegative matrices. In general, $A \in$ $C T N_{n}$ cannot be obtained by $\widehat{A} \in C T N_{n}$ by Example 4.2. Neither can $\widehat{A} \in C T N_{n}$ be obtained by $A \in C T N_{n}$ by Example 4.1.

EXAMPLE 4.3. Let the totally nonnegative matrices $A, B$ be the same as in Example 1.2.

According to [10, Example 2.2], when $\alpha=\{1\}$, we have $A[\alpha \mid \alpha) A(\alpha)^{-1} A(\alpha \mid \alpha]=$ 7.92, and $B[\alpha \mid \alpha) B(\alpha)^{-1} B(\alpha \mid \alpha]=0.98$. Thus

$$
\begin{gathered}
\widehat{A}=\left[\begin{array}{cc}
A[\alpha \mid \alpha) A(\alpha)^{-1} A(\alpha \mid \alpha] & A[\alpha \mid \alpha) \\
A(\alpha \mid \alpha] & A(\alpha)
\end{array}\right]=\left[\begin{array}{ccc}
7.92 & 12 & 13.05 \\
4 & 7 & 8 \\
0 & 2 & 3
\end{array}\right]=\left(\widehat{a}_{i j}\right), \\
\widehat{B}=\left[\begin{array}{cc}
B[\alpha \mid \alpha) B(\alpha)^{-1} B(\alpha \mid \alpha] & B[\alpha \mid \alpha) \\
B(\alpha \mid \alpha] & B(\alpha)
\end{array}\right]=\left[\begin{array}{ccc}
0.98 & 1 & 0 \\
21 & 24 & 14 \\
90.3 & 105 & 70
\end{array}\right]=\left(\widehat{b}_{i j}\right) .
\end{gathered}
$$

Furthermore, using (4.1) and (4.2), it follows that

$$
\begin{gathered}
\widehat{a}_{11} \widehat{a}_{22} \widehat{a}_{33}+\widehat{a}_{31} \widehat{a}_{12} \widehat{a}_{23}=166.32<270.72=\widehat{a}_{11} \widehat{a}_{23} \widehat{a}_{32}+\widehat{a}_{21} \widehat{a}_{12} \widehat{a}_{33}, \\
\widehat{b}_{11} \widehat{b}_{22} \widehat{b}_{33}+\widehat{b}_{21} \widehat{b}_{13} \widehat{b}_{32}=1646.4<2910.6=\widehat{b}_{11} \widehat{b}_{23} \widehat{b}_{32}+\widehat{b}_{21} \widehat{b}_{12} \widehat{b}_{33} .
\end{gathered}
$$

Thus we have $\widehat{A} \notin C T N_{n}$ and $\widehat{B} \notin C T N_{n}$ by Lemma 2.5. 
Example 4.3 reveals that the reason that the inequality (1.3) does not hold for totally nonnegative matrices in [10, Example 2.2] lies in it not satisfying the condition " $\widehat{A} \in C T N_{n}$ or $\widehat{B} \in C T N_{n}$ ". Examples 4.1-4.3 show the condition " $\widehat{A} \in C T N_{n}$ or $\widehat{B} \in C T N_{n}$ " for $A, B \in T N_{n}$ in Theorems $3.1-3.3$ are reasonable in our paper.

Acknowledgements. The authors are grateful to referees for their helpful comments and suggestions to our manuscript.

\section{REFERENCES}

[1] T. Ando. Totally positive matrices. Linear Algebra and its Applications, 90:165-219, 1987.

[2] A.S. Crans, S.M. Fallat, and C.R. Johnson. The Hadamard core of the totally nonnegative matrices. Linear Algebra and its Applications, 328:203-222, 2001.

[3] S.M. Fallat and C.R. Johnson. Hadamard duals, retractbility and Oppenheim's inequality. $O p$ erators and Matrices, 1:369-383, 2007.

[4] S.M. Fallat and C.R. Johnson. Hadamard powers and totally positive matrices. Linear Algebra and its Applications, 423:420-427, 2007.

[5] S. Friedland and E. Virnik. Nonnegativity of Schur complements of nonnegative idempotent matrices. Electronic Journal of Linear Algebra, 17:426-435, 2008.

[6] M. Gasca and J.M. Pena. On factorizations of totally positive matrices. Total Positivity and Its Application. Mathematics and Its Applications, 359, Kluwer Academic Publishing, Dordecht, 109-130, 1996

[7] R.A. Horn and C.R. Johnson. Matrix Analysis. Cambridge University Press, New York, 1985.

[8] C.R. Johnson. Closure properties of certain positivity classes of matrices under various algebra operations. Linear Algebra and its Applications, 97:243-247, 1987.

[9] T.L. Markham. A semigroup of totally nonnegative matrices. Linear Algebra and its Applications, 3:157-164, 1970.

[10] T.L. Markham and R.L. Smith. A Schur complement inequality for certain P-matrices. Linear Algebra and its Applications, 281:33-41, 1998.

[11] B.-Y. Wang and F. Zhang. Schur complements and matrix inequalities of Hadamard product. Linear and Multilinear Algebra, 43(1):315-326, 1997.

[12] B.-Y. Wang, X.-P. Zhang, and F. Zhang. Some inequalities on generalized Schur complements. Linear Algebra and its Applications, 302/303:163-172, 1999.

[13] Z. Yang, S. Liu, and G. Trenkler. Further inequalities involving the Khatri-Rao product. Linear Algebra and its Applications, 430:2696-2704, 2009.

[14] Z. Yang and X. Feng. New lower bound of the determinant for Hadamard product on some totally nonnegative matrices. Journal of Applied Mathematics and Computing, 25:169-181, 2007.

[15] F. Zhang. A matrix identity on the Schur complement. Linear and Multilinear Algebra, 52:367373,2004

[16] F. Zhang (editor). The Schur Complement and its Applications. Numerical Methods and Algorithms, Springer-Verlag, New York, 2005. 